\title{
RESEARCH HIGHLIGHT Anti-Zika virus RNAi in neural progenitor cells
}

\author{
Xuping $\mathrm{Xie}^{1}$ and Pei-Yong Shi ${ }^{1}$ \\ Cell Research (2019) 29:261-262; https://doi.org/10.1038/s41422-019-0158-3
}

\begin{abstract}
Zika virus (ZIKV), a flavivirus that is primarily transmitted by mosquitoes, can cause devastating microcephaly and other congenital syndromes in infants born to infected pregnant women. In a recent paper in Cell Research, $\mathrm{Xu}$ et al. present data demonstrating that (i) ZIKV infection induces RNA interference (RNAi)-mediated antiviral defense in human neural progenitor cells and (ii) treatment with enoxacin, a broad-spectrum antibiotic with known RNAi-enhancing activity, prevents ZIKV infection and microcephalic defect in brain organoids.
\end{abstract}

Zika virus (ZIKV) has recently emerged as a global public health threat. Due to the explosive epidemics and teratogenic potential, the World Health Organization declared ZIKV to be a Public Health Emergency of International Concern in 2016. Besides ZIKV, many other flaviviruses are also significant human pathogens, including dengue, yellow fever, West Nile, Japanese encephalitis, and tickborne encephalitis viruses. Compared with other flaviviruses, ZIKV is unique in causing congenital syndrome in fetuses of infected pregnant women, including microcephaly, craniofacial disproportion, ocular abnormalities, spasticity, and miscarriage. Adults infected with ZIKV may develop Guillain-Barré syndrome, an autoimmune disorder caused by the immune system damaging the peripheral nervous system. The U.S. Centers for Disease Control and Prevention estimated one in seven infants born to mothers infected during pregnancy may develop birth defects. ${ }^{1}$ Upon maternal-to-fetal transmission in pregnant women, ZIKV preferentially infects human neural progenitor cells (hNPCs) in the fetal brain. ${ }^{2}$ The infection impairs hNPC proliferation and differentiation, triggers apoptosis of progeny cells, and debilitates brain development. ${ }^{3}$ Thus, it is critical to define the immune response when hNPCs are infected by ZIKV. Such knowledge is critical to understand ZIKV-mediated congenital syndrome as well as to develop novel countermeasures.

All flaviviruses produce double-stranded RNA (dsRNA) as a replication intermediate during infection. The dsRNA serves as a pathogen-associated molecular pattern (PAMP) to elicit interferon (IFN)-mediated antiviral defense in mammalian host. Although RNA interference (RNAi) has been well established as an evolutionarily conserved innate immune response in fungi, plants, and invertebrates, its role in antiviral defense in mammalians remains to be fully established. ${ }^{4}$ To achieve this goal, $\mathrm{Xu}$ and colleagues have now provided three sets of compelling evidence supporting that ZIKV infection induces RNAi-mediated antiviral response in hNPCs. ${ }^{5}$

First, Xu et al. ${ }^{5}$ demonstrated an abundant production of virusderived small interfering RNAs (vsiRNAs) in ZIKV-infected hNPCs (Fig. 1a). vsiRNAs were measured by deep sequencing and validated by northern blot. Approximately $63 \%$ of the virus- derived small RNA (vsRNA) reads were 21-23 nt in length, with predominant 22-nt vsRNAs representing both positive- and negative-strand RNAs. However, once hNPCs had been differentiated into neurons, they lost the ability to produce vsiRNAs after ZIKV infection. This result suggests a switch-off of the vsiRNA biogenesis pathway during the hNPC-to-neuron differentiation. The conclusion is further supported by the results that no vsiRNA was detected in ZIKV-infected human rhabdomyoma RD cells or astrocytes. ${ }^{6}$ Altogether, the data indicate that hNPCs represent a unique cell population that has a functional RNAi machinery to process viral dsRNAs to vsiRNAs. These vsiRNAs are then loaded onto the RNA-induced silencing complexes (RISCs) to initiate the cleavage of viral RNA in infected hNPCs (Fig. 1a).

Second, $\mathrm{Xu}$ et al. showed that the production of vsiRNAs is dependent on the authentic RNAi pathway in ZIKV-infected hNPCs (Fig. 1a). Knockdown or knockout of Dicer or AGO2, the two essential components of RNAi pathway, significantly enhanced ZIKV replication in hNPCs. In addition, ectopic expression of the Nodamura virus B2 protein, a known viral suppressor of RNAi (VSR), increased ZIKV replication in hNPCs. These results demonstrate that (i) an intact RNAi pathway is required for the production of vsiRNAs in ZIKV-infected hNPCs and (ii) the vsiRNAs are functional in inhibiting ZIKV infection.

Third, Xu et al. evinced a therapeutic approach using an RNAi enhancer to treat ZIKV infection in hNPCs and brain organoids (Fig. 1b). Enoxacin is a clinically approved antibiotic with known RNAi-enhancing activity, possibly through modulating PIWIL3 (piwi like RNA-mediated gene silencing 3, an Argonaute protein of the PIWI subfamily). ${ }^{7}$ In hNPCs, treatment with enoxacin suppressed ZIKV infection with $\mathrm{IC}_{50}$ of $52 \mu \mathrm{M}$ and $\mathrm{CC}_{50}$ of $176 \mu \mathrm{M}$. However, the compound failed to inhibit ZIKV in Dicerknockout hNPCs, indicating that the observed antiviral activity is dependent on the RNAi pathway. In brain organoids, enoxacin inhibited ZIKV infection of hNPCs in the ventricular zone (VZ) and subventricular zone (SVZ), rescued hNPC proliferation, and maintained a normal brain development. These promising results warrant further in vivo efficacy testing of enoxacin in mouse or non-human primate microcephaly models.

The findings by Xu et al. have opened new directions for future research. First, IFN pathway has been well established as the major innate immunity in mammalians. However, recent studies indicate that stem cells are refractory to IFN, but intrinsically express a subset of IFN-stimulated genes (ISGs) for antiviral defense. ${ }^{8}$ The discovery of functional vsiRNAs in ZIKV-infected hNPCs suggests that, together with distinct ISGs in stem cells, RNAi may serve as another arm of antiviral defense. It remains to be determined (i) whether the results observed in hNPCs could be generalized to stem cells of other tissue types and (ii) whether other viruses

\footnotetext{
'Department of Biochemistry and Molecular Biology, Sealy Center for Structural Biology and Molecular Biophysics, Sealy Institute for Vaccine Sciences, University of Texas Medical Branch, Galveston, Texas 77555, USA

Correspondence: Pei-Yong Shi (peshi@utmb.edu)
}

Published online: 15 March 2019 


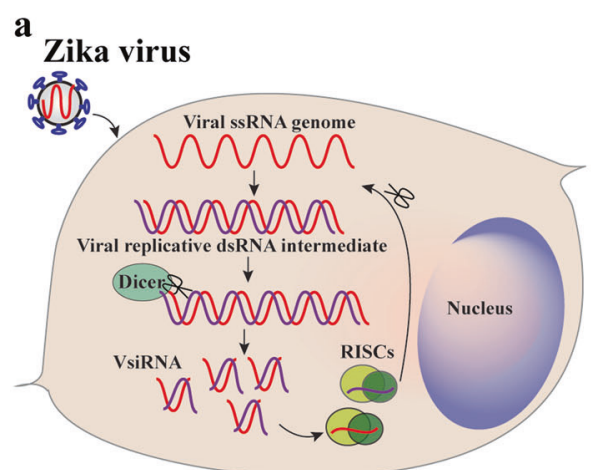

Human neural progenitor cell

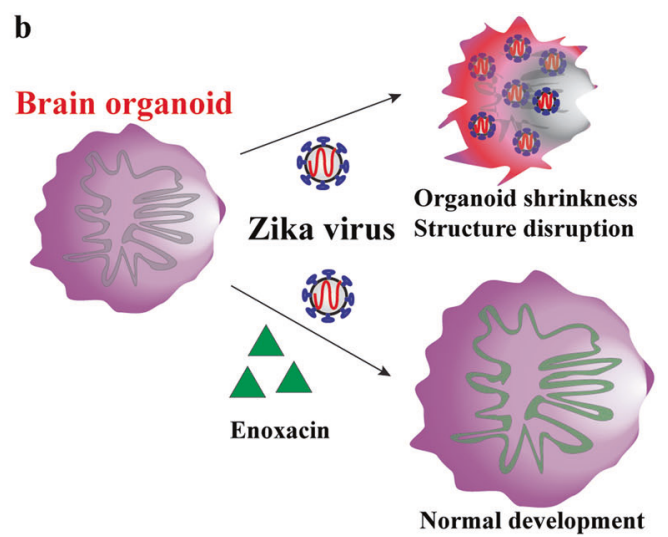

Fig. 1 RNAi-mediated antiviral defense in hNPCs (a) and brain organoids (b). a In ZIKV-infected hNPCs, viral dsRNA is processed by Dicer to generate vsiRNAs that are loaded onto RISCs to cleave viral RNA. b Enoxacin rescues the normal development of ZIKV-infected brain organoids. Without enoxacin treatment, ZIKV infection impairs the development of brain organoids. With enoxacin treatment, the brain organoids are protected against ZIKV infection and their normal development is maintained

behave the same as ZIKV in hNPCs. Second, the importance of RNAi as a mammalian antiviral defense mechanism remains controversial. ${ }^{4}$ Qiu et al. recently showed that the lack of detection of vsiRNA is likely due to the expression of potent VSRs that completely block vsiRNA biogenesis. ${ }^{9}$ Notably, previous studies were performed in somatic cells, not stem cells. Thus, it is not surprising that hNPCs lost their ability to produce vsiRNAs after they had differentiated into neurons. ${ }^{5}$ Future experiments are needed to identify the molecular switch that is responsible for the shutdown of vsiRNA biogenesis during the hNPC-to-neuron differentiation. Third, RNAi is the major innate immune pathway in mosquitoes to control arbovirus infection and transmission. Since ZIKV is transmitted by mosquitoes, the virus is expected to develop VSRs to overcome RNAi suppression of viral replication in insects. Indeed, flavivirus capsid protein was previously shown to bind to dsRNA and suppress RNAi. ${ }^{10}$ It remains to be determined (i) whether the capsid protein dampens the RNAi-mediated antiviral defense in ZIKV-infected hNPCs and (ii) whether evolutionary mutations in ZIKV capsid protein affect the potency of RNAi suppression, leading to changed efficiency in mosquito infection and microcephaly development. Collectively, the study by $\mathrm{Xu}$ et al. represents a major advance in elucidating ZIKV-host interactions in hNPCs and supports RNAi as an antiviral defense mechanism in mammalian host.

\section{ADDITIONAL INFORMATION}

Competing interests: The authors declare that there is no conflict of interest.

\section{REFERENCES}

1. Rice, M. E. et al. MMWR Morb. Mortal. Wkly Rep. 67, 858-867 (2018).

2. Tang, H. et al. Cell Stem Cell 18, 587-590 (2016).

3. Qian, X. et al. Cell 165, 1238-1254 (2016).

4. Cullen, B. R. Immunity 46, 970-972 (2017).

5. Xu, Y.-P. et al. Cell Res. 2019. https://doi.org/10.1038/s41422-019-0152-9

6. Kozak, R. A. et al. Viruses 9, pii: E297 (2017).

7. Abell, N. S., Mercado, M., Caneque, T., Rodriguez, R. \& Xhemalce, B. J. Am. Chem. Soc. 139, 1400-1403 (2017).

8. Wu, X. et al. Cell 172, 423-438 (2018).

9. Qiu, Y. et al. Immunity 46, 992-1004 (2017).

10. Samuel, G. H., Wiley, M. R., Badawi, A., Adelman, Z. N. \& Myles, K. M. Proc. Natl. Acad. Sci. USA 113, 13863-13868 (2016). 